\title{
A New Method to Evaluate Equity-Linked Life Insurance
}

\author{
Ming-Shann Tsai \\ National University of Kaohsiung \\ E-Mail: mstsai@nuk.edu.tw \\ Shih-Cheng Lee \\ Yuan-Ze University \\ University of Adelaide \\ E-Mail: sclee@saturn.yzu.edu.tw \\ Jiun-Lin Chen \\ University of Adelaide \\ E-Mail: alex.chen@adelaide.edu.au \\ Shu-Ling $\mathrm{Wu}$ \\ Nan Shan Life Insurance Co., Ltd \\ E-Mail: bbkoala0712@nanshanlife.com.tw
}

\begin{abstract}
This paper provides a new method to appraise equity-linked life insurance products. Using the mixture mortality model to determine the death and survival probability, we calculate the premium for a death claim and a survival claim for equity-linked life insurance policies. The numerical result shows that the premium is higher than that calculated using the Brennan and Schwartz model. As a mixed survival function can solve the problems of the rigid application in Life Tables, the premium from our method is more accurate for life insurance. Thus, our method can help life insurance companies avoid potential losses from mispriced products.
\end{abstract}

Keywords: Equity-Linked Life Insurance, Mixture Mortality Model, Mortality Rate 


\section{INTRODUCTION}

Life insurance has prevailed for more than 100 years. Traditional insurance provides only constant benefits when the insured person dies. However, because of the tremendous development of financial markets in recent decades, life insurance is designed not only to provide life protection, but also to represent an important investment contract to satisfy the needs of investors. For example, the most famous innovative life insurance product is called equity-linked life insurance. To appraise such insurance, two sources of risk must be considered: the payoff uncertainty and the time uncertainty. As the payoff of an equity-linked contract with a minimum guarantee is just like an option, researchers always use option pricing theory to solve the problem of payoff uncertainty (see Brennan and Schwartz, 1976 \& 1979; Bacinello and Ortu, 1993; Grosen and Jorgensen, 1997; Persson and Aase, 1997; Nonnenmacher and Rub, 1998; Bacinello, 2003). Meanwhile, scholars always use Life Tables to solve the problem of time uncertainty and determine the premium for insurance.

Because of medical technology advances and the decreasing birth rate, the dependency ratio has gradually grown throughout the world. This ratio is also expected to rise progressively for the foreseeable future. Therefore, it is incorrect to evaluate life insurance products using the traditional pricing method. Furthermore, the life insurance company experiences losses due to inaccurate pricing results. Two problems arise from obtaining the mortality rate from Life Tables. First, the data in Life Tables only reflect historical mortality information. One cannot obtain a forecasted mortality rate for evaluating the insurance. Second, we cannot obtain mortality information between two ages because Life Tables only present the mortality rate for a discrete period. Thus, we argue that the premium for life insurance might be underestimated using Life Tables. To more accurately estimate the premium, we need to carefully consider how to forecast the mortality rate accurately.

At present, several well-known mortality functions are supported for estimating mortality rates, such as Weibull-type for children, inverse-Weibull-type for teenagers, and Gompertz-type for adults (see Brillinger, 1961; Tenenbein and Vanderhoof, 1980; Wetterstrand, 1981; Lee and Carter, 1992; Carriere, 1992, 1994; and Jasiulewicz, 1997). To the best of our knowledge, no research has combined these mortality models to evaluate the equity-linked life insurance. This paper uses the mixture mortality function reported by Carriere $(1992,1994)$ to obtain the mortality rate. Then, under the assumption of a stochastic interest rate, we obtain the premium for equity-linked life insurance with a minimum guarantee. The advantages of this model are that one can more precisely price an insurance contract using forecasted mortality rate and more clearly analyze the influence of the change of parameters of mortality rate on insurance. 
Therefore, researchers can forecast the change of the parameter of the mortality rate and obtain more accurate premiums for insurance products. To the best of our knowledge, this is the first paper to use the forecasted mortality rate in the valuation of equity-linked life insurance products. As we know, the life insurance system provides important social welfare for a country. Our model is significant because the dependency ratio is gradually growing in the world. If the valuation model for life products ignores this trend, the life insurance company might suffer losses from selling these life products, which can even cause bankruptcy for the company and further influence the social welfare as well as hurt the economic development of a country.

The paper is organized as follows. In Section 2, we describe the models for equity-linked life insurance and the mixture mortality function. In Section 3, we use the open data from Life Tables to estimate the parameters of the mixture mortality model and determine the value of life insurance using a numerical method. Section 4 gives the conclusion.

\section{MODEL}

We define the states on the probability space $(\Omega, \Phi, P)$ as $\Phi_{t}=\Psi_{t} \vee F_{t}$, where $\Omega$ represents state space; $\Psi_{t}$ represents the $\sigma$-field of the death at time $t ; F_{t}$ represents the $\sigma$-field for infiltration of other information on the bond at time $t$; $P$ represents the physic measure; and $Q$ represents the martingale measure. Jarrow, Lando and $\mathrm{Yu}$ (2005) emphasized that the equivalence between the physical and martingale measures holds under the assumption of a well-diversified portfolio. As the mortality risk can be diversified by selling the same kind of contract to enough people, we can obtain the market prices of insurance under the assumption of a well-diversified portfolio. Therefore, the physical measure is identical to the martingale measure. The mortality risks can be calculated from market information. Let $G(t)$ be the survival function at time $t ; F(t)$ is the death probability at time $t$, where $F(t)=1-G(t)$; and $f(t)$ is the

probability density function for death, where $f(t)=\frac{\partial F(t)}{\partial t}$. We assume that the lifetime is independent of the interest rate and portfolio.

The payoff for equity-linked life insurance is dependent on the performance of the portfolio. We denote the initial portfolio value as $y(t)$ at time $t$. Under the martingale measure, the change in portfolio value $y$ can be described as follows:

$$
\frac{d y}{y}=r(t) d t+\sigma_{y} d Z_{y}(t),
$$


where

$\sigma_{y}^{2}$ is the instantaneous variance of portfolio and

$d Z_{y}(t)$ is the standard Brownian motion of portfolio.

The insurer provides a minimum guarantee for equity-linked life insurance. To simplify, we assume the minimum guarantee is equal to a portion $(\theta)$ of the initial portfolio value. The basic idea of pricing a contingent payoff using the risk-neutral probability measure is that one can perfectly replicate the payoff of the contingent claim with a self-financing strategy. When a payoff is contingent on the mortality of the holder, perfect hedging using a self-financing trading strategy becomes impossible because the mortality risk cannot be traded on the financial market. One solution for overcoming this problem is to consider the risk-minimizing hedging strategy (see Lin and Tan, 2003). Hence, the death claim at time $s$ is described as an option. Using the option-pricing theory, the expected present value of the death claim, $R_{p v}(t, s)$, is:

$$
R_{p v}(t, s)=y(t) N\left(d_{1}(s)\right)-B(t, s) \theta y(t) N\left(d_{2}(s)\right)+B(t, s) \theta y(t),
$$

where

$$
d_{1}(s)=\frac{\ln \theta-\ln B(t, s)+0.5 \sigma_{x}^{2}(s-t)}{\sigma_{x} \sqrt{s-t}}
$$

$\sigma_{x}^{2}=\sigma_{y}^{2}+\sigma_{r}^{2}(s)-2 \rho \sigma_{r}(s) \sigma_{y}$ is a component variance of the portfolio and the interest rate,

$\rho$ is the correlation between the portfolio and the interest rate,

$N(\cdot)$ is the cumulative normal distribution function, and

$B(t, s)$ represents the value of a zero coupon bond with maturity date $s$ in time $t$.

It should be noted that the zero coupon bond is defined as $B(t, s)=E\left(e^{-\int_{t}^{s} r(u) d u}\right)$, where $r(u)$ is the stochastic spot interest rate. We can use interest rate models, such as Vasicek's (1977) model and the Cox, Ingersoll, and Ross (1985) model, to describe the value of a zero coupon bond.

The premium for equity-linked life insurance at initial time $t$ using the risk-minimizing hedging strategy is described as follows:

$$
D_{t}=R_{p v}(t, T) G(T \mid t)+\int_{t}^{T} R_{p v}(t, s) f(s \mid t) d s,
$$

where

$G(T \mid t)$ is the survival probability at time $T$ conditional on time $t$, and 
$f(s \mid t)$ is the death probability density function at time $s$ conditional on time $t$.

The mixture survival model is a mixture of three distinct distributions: a Weibull distribution for childhood mortality, an inverse Weibull distribution for teenage mortality, and a Gompertz distribution for adulthood mortality. The mixture survival model can be specified as follows:

$$
G(t)=\sum_{i=1}^{3} \psi_{i} G_{i}(t)
$$

where

$\psi_{i}$ is the probability of different periods, and $\psi_{1}+\psi_{2}+\psi_{3}=1$; and

$G_{i}(t)$ is a specific survival function, $i=1,2,3$, which represents the functions of Weibull, inverse-Weibull, and Gompertz, respectively.

The survival probability density function of Weibull, inverse-Weibull, and Gompertz are described as follows:

$$
\begin{aligned}
& G_{1}(t)=\exp \left(-\left(\frac{t}{m_{1}}\right)^{\frac{m_{1}}{\sigma_{1}}}\right), \\
& G_{2}(t)=1-\exp \left(-\left(\frac{t}{m_{2}}\right)^{-\frac{m_{2}}{\sigma_{2}}}\right), \text { and } \\
& G_{3}(t)=\exp \left(\exp \left(-\frac{m_{3}}{\sigma_{3}}\right)-\exp \left(\frac{t-m_{3}}{\sigma_{3}}\right)\right),
\end{aligned}
$$

where $m_{i}$ and $\sigma_{i}, i=1,2,3$, are the location parameters and dispersion parameters. Therefore, the death probability density function is described as follows:

$$
f(t)=\frac{\partial(1-G(t))}{\partial t}=\sum_{i=1}^{3} \psi_{i} f_{i}(t)
$$

where

$$
\begin{aligned}
& f_{1}(t)=\frac{1}{\sigma_{1}}\left(\frac{t}{m_{1}}\right)^{\frac{m_{1}}{\sigma_{1}}-1} \exp \left(-\left(\frac{t}{m_{1}}\right)^{\frac{m_{1}}{\sigma_{1}}}\right), \\
& f_{2}(t)=\frac{1}{\sigma_{2}}\left(\frac{t}{m_{2}}\right)^{-\frac{m_{2}}{\sigma_{2}}-1} \exp \left(-\left(\frac{t}{m_{2}}\right)^{-\frac{m_{2}}{\sigma_{2}}}\right) \text {, and }
\end{aligned}
$$




$$
f_{3}(t)=\frac{1}{\sigma_{3}} \exp \left(\frac{t-m_{3}}{\sigma_{3}}+\exp \left(-\frac{m_{3}}{\sigma_{3}}\right)-\exp \left(\frac{t-m_{3}}{\sigma_{3}}\right)\right)
$$

Considering that the insured is alive at time $t$, the conditional survival probability density function and conditional death probability density function are described as follows:

$$
\begin{aligned}
& G(s \mid t)=\frac{G(s)}{G(t)} \\
& f(s \mid t)=\frac{f(s)}{G(t)} .
\end{aligned}
$$

\section{NUMERICAL ANALYSIS}

We used the Life Tables of the USA and Taiwan in the year 2005 to estimate the parameters of the survival function. The data were obtained from the Human Life-Table Database (http://www.lifetable.de). We compared the premiums in our model with the model used by Brennan and Schwartz (1976). The estimated parameters for males are presented in Table 1.

Table 1 Parameter Estimates for the Mixture Mortality Model

\begin{tabular}{cccccccccc}
\hline Country & $\sigma_{1}$ & $m_{1}$ & $\sigma_{2}$ & $m_{2}$ & $\sigma_{3}$ & $m_{3}$ & $\psi_{1}$ & $\psi_{2}$ & $\psi_{3}$ \\
\hline USA & 32.697 & 2.869 & 7.468 & 52.427 & 11.595 & 81.17 & 0.0126 & 0.000001 & 0.9874 \\
Taiwan & 2.7065 & 1.294 & 4.071 & 52.931 & 12.959 & 79.86 & 0.0095 & 0.000001 & 0.9905 \\
\hline
\end{tabular}

We made the following assumptions: The survival claim and death claim are both $\$ 100,000$; the age of the insured is 35 years old; the variance of the portfolio is $20 \%$; and the ratio of participation is equal to 1 . In addition, for simplicity, we let $\rho=0$ and obtained the zero coupon bond under a constant spot interest rate (i.e., 2.5\%). The calculated values of insurance premiums are presented in Table 2, which shows that the premium for a death claim is higher in our model. Moreover, the total premium calculated from our model is higher than that calculated from the Brennan and Schwartz model. Therefore, the premium for life insurance might be underestimated using the traditional method with Life Tables. 
Table 2 Comparison of Proposed Model with Brennan and Schwartz's (1976) Model for USA and Taiwan Data

\begin{tabular}{crrrrrrr}
\hline & & \multicolumn{3}{c}{ Our model } & \multicolumn{3}{c}{ Brennan and Schwartz (1976) } \\
\hline \multirow{4}{*}{ USA } & Age & \multicolumn{2}{c}{ Total } & Survival & Dead & Total & \multicolumn{2}{c}{ Survival } & \multicolumn{1}{c}{ Dead } \\
premium & premium & premium & premium & premium & premium \\
& 25 & 112,338 & 107,910 & 4,428 & 111,910 & 107,180 & 4,730 \\
& 35 & 113,046 & 102,700 & 10,346 & 111,923 & 102,430 & 9,493 \\
& 45 & 114,685 & 91,337 & 23,348 & 111,927 & 9,111 & 20,816 \\
\hline \multirow{2}{*}{ Taiwan } & 25 & 112,536 & 106,080 & 6,456 & 111,911 & 105,910 & 6,001 \\
& 35 & 113,438 & 99,705 & 13,733 & 111,923 & 100,070 & 11,853 \\
& 45 & 115,300 & 87,178 & 28,122 & 111,923 & 88,431 & 23,492 \\
\hline
\end{tabular}

\section{CONCLUSION}

A fair premium will encourage people to maintain life insurance. This paper constructs a model to evaluate the fair price for equity-linked life insurance. Compared with traditional research methods, we adopted a mixture model of the Weibull function, inverse-Weibull function, and Gompertz survival function to fit the pattern of mortality rates in Life Tables. We then used the parameters estimated to calculate the survival probability and death probability. Finally, we determined the premiums for endowment insurance and equity-linked life insurance. The total premium calculated using our model is higher than that calculated using the Brennan and Schwartz model. We believe that using a mixed survival function can solve the problems of the rigid application in Life Tables. Thus, this method gives a more accurate premium for life insurance. From the managerial viewpoint, our model can not only help the life insurance company avoid losses from inaccurate valuations of an equity-linked life insurance product, but also assist the risk manager of the life insurance company to efficiently control the mortality risk related with this product. Because we found the total premium calculated from our model to be higher than that calculated from the Brennan and Schwartz model, we also suggest that stakeholders can raise the current premiums to avoid losses stemming from the evaluation of the premiums of related life products using traditional models. 


\section{REFERENCES}

Bacinello, A.R., and Ortu, F. (1993a). Pricing equity-linked life insurance with endogenous minimum guarantees. Insurance: Mathematics and Economics, 12, 245-257. http://dx.doi.org/10.1016/0167-6687(93)90411-H

Bacinello, A.R. (2003). Fair valuation of a guaranteed life insurance participating contract embedding a surrender option. The Journal of Risk and Insurance, 70, 461-487. http://dx.doi.org/10.1111/1539-6975.t01-1-00060

Brennan, M.J., and Schwartz, E.S. (1976). The pricing of equity-linked life insurance policy with an asset value guarantee. Journal of Financial Economics, 3, 195-213. http://dx.doi.org/10.1016/0304-405X(76)90003-9

Brennan, M.J., and Schwartz, E.S. (1979). Alternative investment strategies for the issues of equity-linked life insurance policies with an asset value guarantee. Journal of Business, 52, 63-93.

Brillinger, D.R. (1961). A justification of some common laws of mortality. Transactions of the Society of Actuaries, 13, 116-126.

Carriere, J.F. (1992). Parametric models for life tables. Transactions of the Society of Actuaries, 44, 77-99.

Carriere, J.F. (1994). A select and ultimate parametric model. Transactions of the Society of Actuaries, 46, 75-97.

Cox, J.C., Ingersoll, J.E., and Ross, S.A. (1985). A theory of the term structure of interest rates. Econometrica, 53, 385-407. http://dx.doi.org/10.2307/1911242

Grosen, A., and Jorgensen, P.L. (1997). Valuation of early exercisable interest rate guarantees. The Journal of Risk and Insurance, 64, 481-503. http://dx.doi.org/10.2307/253761

Jarrow, R.A., Lando, D., and Yu, F. (2005). Default risk and diversification: theory and empirical implications. Mathematical Finance, 15, 1-26. http://dx.doi.org/10.1111/j.0960-1627.2005.00208.x

Jasiulewicz, H. (1997). Application of mixture models to approximation of age-at-death distribution. Insurance: Mathematics and Economics, 19, 237-241. http://dx.doi.org/10.1016/S0167-6687(96)00014-5

Lee, R.D., and Carter, L.R. (1992). Modeling and forecasting U.S. mortality. Journal of the American Statistical Association, 87, 659-671. http://dx.doi.org/10.2307/2290201

Lin, X.S., and Tan, K.S. (2003). Valuation of equity-indexed annuities under stochastic interest rates. North American Actuarial Journal, 7, 72-91.

Nonnenmacher, D.J.F., and Rub, J. (1998). Risk models in the context of equity-linked life insurance contracts. The Geneva Papers on Risk and Insurance Theory, 21, 
65-102.

Persson, S.A., and Aase, K.K. (1997). Valuation of the minimum guaranteed return embedded in life insurance products. Journal of Risk and Insurance, 64, 599-618. http://dx.doi.org/10.2307/253888

Tenenbein, A., and Vanderhoof, I.T. (1980). New mathematical laws of select and ultimate mortality. Transactions of the Society of Actuaries, 32, 119-183.

Vasicek, O.A. (1977). An equilibrium characterization of the term structure. Journal of Financial Economics, 5, 177-188. http://dx.doi.org/10.1016/0304-405X(77)90016-2 Wetterstrand, W.H. (1981). Parametric models for life insurance mortality data: Gompertz's law over time. Transactions of the Society of Actuaries, 33, 159-179. 
